University of Nebraska - Lincoln

DigitalCommons@University of Nebraska - Lincoln

3-1-2007

\title{
Molecular dynamics of homogeneous nucleation in the vapor phase of Lennard-Jones. III. Effect of carrier gas pressure
}

Kenji Yasuoka

University of Nebraska-Lincoln

Xiao Cheng Zeng

University of Nebraska-Lincoln, xzeng1@unl.edu

Part of the Chemistry Commons

Yasuoka, Kenji and Zeng, Xiao Cheng, "Molecular dynamics of homogeneous nucleation in the vapor phase of Lennard-Jones. III. Effect of carrier gas pressure" (2007). Xiao Cheng Zeng Publications. 1. https://digitalcommons.unl.edu/chemzeng/1

This Article is brought to you for free and open access by the Published Research - Department of Chemistry at DigitalCommons@University of Nebraska - Lincoln. It has been accepted for inclusion in Xiao Cheng Zeng Publications by an authorized administrator of DigitalCommons@University of Nebraska - Lincoln. 


\title{
Molecular dynamics of homogeneous nucleation in the vapor phase of Lennard-Jones. III. Effect of carrier gas pressure
}

\author{
Kenji Yasuoka \\ Department of Mechanical Engineering, Keio University, Yokohama 223-8522, Japan \\ and Department of Chemistry, University of Nebraska-Lincoln, Lincoln, Nebraska 68588 \\ X. C. Zeng \\ Department of Chemistry, University of Nebraska-Lincoln, Lincoln, Nebraska 68588 \\ (Received 2 January 2007; accepted 5 February 2007; published online 30 March 2007)
}

\begin{abstract}
A molecular dynamics simulation of vapor phase nucleation has been performed with 40000 Lennard-Jones particles for the target gas and 0-160 000 particles for the carrier gas. Three carrier gas models are adopted, including a soft-core model, a Lennard-Jones model, and a modified Lennard-Jones model in which the attractive interaction can be adjusted. The effect of the carrier-gas pressure is assessed through computing and comparing the rate of nucleation and cluster size distribution. It is found that the effect of the carrier-gas pressure can be strongly dependent on the carrier-gas model. A positive effect (enhancement of the nucleation rate) is found with the soft-core potential model, whereas negligible effect is found with the Lennard-Jones potential model. For the modified Lennard-Jones potential with a weak attractive interaction, the carrier-gas effect is positive. However, the effect is negligible with a stronger attractive interaction between the target and carrier-gas particles. A reason for the negligible effect is that the carrier-gas particles are adsorbed on the cluster surface when the density of target and carrier-gas particles are comparable. When the density of carrier-gas particles are four times that of the target particles, the carrier-gas particles tend to mix with the target particles in the clusters. () 2007 American Institute of Physics. [DOI: $10.1063 / 1.2712436]$
\end{abstract}

\section{INTRODUCTION}

In vapor to liquid nucleation experiments, the vapor to be studied (called the target gas) are mixed with a background gas, namely, the carrier gas. The latter is a supercritical component of the vapor mixture. Because the temperature is usually set up above its critical temperature, the carrier gas is expected to show no tendency of condensation by itself. Therefore, it is generally assumed that the role of the carrier gas is purely for thermalization of embryonic clusters of the target gas. If the carrier gas was absent, the latent heat of condensation could not be easily removed from newly formed clusters, which would result in overheating of the clusters. As such, the condensation or nucleation process would proceed not in the isothermal condition. A question arises that if the carrier-gas pressure increases substantially would the carrier-gas strongly affect the nucleation of the target gas in addition to the thermalization of the clusters? Previous experiments with the diffusion cloud chamber ${ }^{1-5}$ showed that the effect of the carrier gas on nucleation becomes stronger with decreasing temperature and increasing the molecular weight of the target gas. However, the effect of the carrier-gas pressure on nucleation was not observed in previous experiments with the expansion cloud chambers. ${ }^{6-9}$ Moreover, in some experiments the effect of the carrier gas pressure was shown to be negative, ${ }^{1-5,10-12}$ that is, the carrier gas reduced the rate of nucleation, while in some other experiments the effect was shown to be positive. ${ }^{13-15}$ The ap- parent conflicting results from different experiments called for more molecular-level investigations of the carrier-gas effect.

Molecular simulation is an ideal tool to study the carriergas effect for model molecular systems. Oh and one of the authors ${ }^{16}$ performed Monte Carlo simulation to compute the formation free energy of water clusters in the presence of a nitrogen carrier gas. They found that the carrier-gas pressure has a negative effect on water nucleation at low temperatures. Tanaka et $a l .{ }^{17}$ reported a negligible effect of the carrier-gas pressure on the cluster size distribution. They used a Lennard-Jones system for the target gas and compared the cluster size distribution with a soft-core carrier gas and without carrier gas. Tang and Ford ${ }^{18}$ carried out molecular dynamics (MD) simulation to study the evaporation dynamics of an Ar cluster in the presence of a He carrier gas. The potential model for Ar and He was the Lennard-Jones potential. They reported that the effect of the carrier-gas pressure on the evaporation dynamics of the Ar cluster is negligible. Toxvaerd ${ }^{19}$ also performed a MD simulation for a LennardJones (LJ) system in the presence of a model carrier gas described by repulsive LJ potential. He concluded that the carrier-gas particles were squeezed out of the target clusters before the onset of nucleation even the number of carrier-gas particles is the dominant component in the vapor mixture.

In the two previous papers of this series (papers I and II), ${ }^{20,21}$ we reported MD simulations of homogeneous nucleation processes in a supersaturated $\mathrm{LJ}$ and water vapor, respectively. In particular, we introduced a simulation method 
to compute the rate of homogeneous nucleation in the steady state. In paper I, we used a repulsive Lennard-Jones model for the carrier gas. In this paper, we considered three models of the carrier gas based on the Lennard-Jones potential. Particular attention is placed on the effect of the carrier-gas pressure on the rate of nucleation in the steady state. We found that indeed the effect of the carrier-gas pressure can be sensitive to the carrier-gas model. Only positive or negligible effects of the carrier gas were observed in the MD simulations with the three models of the carrier gas.

\section{MODEL AND METHOD}

The simulation system was a vapor mixture consisting of 40000 target particles and 0-160000 carrier-gas particles, similar to that used in paper $\mathrm{I}^{20}$ The interaction potential between target particles was a LJ type, i.e.,

$$
U_{\mathrm{tt}}(r)=4 \varepsilon_{\mathrm{tt}}\left[\left(\frac{\sigma}{r}\right)^{12}-\left(\frac{\sigma}{r}\right)^{6}\right],
$$

where $r$ was the interatomic distance. The LJ parameter for the target-target particle interaction was set to be $\varepsilon_{\mathrm{tt}}=\varepsilon$.

Three carrier-gas models were adopted, including a softcore model (model A), a Lennard-Jones model (model B), and a modified Lennard-Jones model (model C) in which the attractive interaction can be adjusted. Model A is purely a soft-core type without an attractive interaction as used in paper $\mathrm{I}^{20}$

$$
U_{\mathrm{cc}}(r)=U_{\mathrm{tc}}(r)=4 \varepsilon\left[\left(\frac{\sigma}{r}\right)^{12}\right] .
$$

This potential was used for both targe-carrier and carriercarrier interactions. For model B we considered two different values of the LJ potential parameter for the interaction between carrier-carrier particles: $\varepsilon_{\mathrm{cc}}=0.1 \varepsilon$ and $\varepsilon_{\mathrm{cc}}=0.25 \varepsilon$. A mixing rule $\varepsilon_{\mathrm{tc}}=\sqrt{\varepsilon_{\mathrm{tt}} \varepsilon_{\mathrm{cc}}}$ was applied to obtain the corresponding LJ parameter for the target-carrier interaction, $\varepsilon_{\mathrm{tc}}$ $=0.316 \varepsilon$ and $0.5 \varepsilon$. Model $\mathrm{C}$ is a modified LJ potential,

$$
U_{\mathrm{cc}}(r)=U_{\mathrm{tc}}(r)=4 \varepsilon\left[\left(\frac{\sigma}{r}\right)^{12}-k\left(\frac{\sigma}{r}\right)^{6}\right],
$$

where $k$ is an adjustable parameter with values between 0 and 1 . The $k$ parameter was used to change the attractive interaction. If $k=0$, model $\mathrm{C}$ is purely a soft-core potential, the same as model $\mathrm{A}$; if $k=1$, model $\mathrm{C}$ represents a LJ potential, the same as model $\mathrm{B}$. We selected three values of $k$ to be $0.1,0.3$, and 0.5 for model $\mathrm{C}$. The $\mathrm{LJ}$ potential parameter $\sigma$ and the mass $m$ for all target and carrier-gas particles were set to be identical in all simulations. Hereafter, $\varepsilon, \sigma$, and $m$ are used as the units of energy, length, and mass, respectively.

The simulated vapor condition was set the same as that in paper $\mathrm{I}^{20}$ At the initial state, the number density of target particles was set to be $2.315 \times 10^{-2}$. The simulation cell size was $120 \times 120 \times 120$ in the dimensionless unit. The cutoff radius for the interatomic interaction was 4.5 . No tail correction was made for the energy calculation because of the spatial inhomogeneity in the nucleation process. In the MD simulation, the entire system was initially equilibrated for

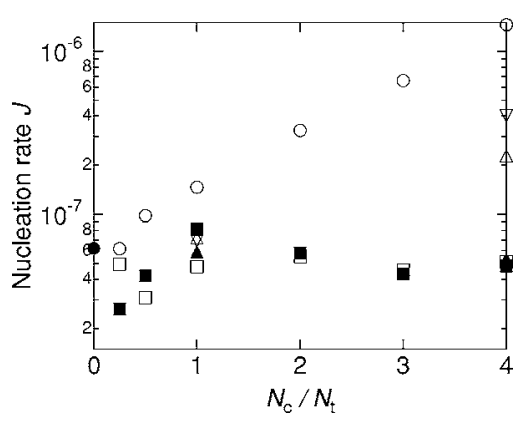

FIG. 1. The nucleation rate vs the ratio of the number of carrier-gas particles to the number of target particles with various models of the carrier gas: $(\bigcirc)$, model A, model B, $(\square) \varepsilon_{\mathrm{cc}}=0.1$ and $(\square) \varepsilon_{\mathrm{cc}}=0.25$, model C, $(\nabla) k=0.1,(\triangle)$ $k=0.3$, ( $\mathbf{\Delta}) k=0.5$ and $(\mathbf{O})$ no carrier gas.

50000 time steps at $T=2.0$ and then quenched to the state $T=0.67$, which was near the triple-point temperature of the target gas. Subsequent to the quench, a total of 300000 time step (or 1500 in dimensionless units) MD runs were carried out. Each time step was 0.005 . The temperature of the carrier gas was controlled by using the Nosé-Hoover method. In the simulation without the carrier gas, the temperature of the target gas was controlled by the Nosé-Hoover method. For the analysis of MD results (see below), trajectories of the target particles were recorded to examine the effect of the carrier-gas pressure to the nucleation of the cluster.

\section{RESULTS AND DISCUSSIONS}

\section{A. Nucleation rate}

The nucleation rate $J$ is defined as the number of nuclei (larger than the critical nucleus) generated per unit volume per unit time. To estimate the nucleation rate at the steady state we employed the same method introduced in papers I and II. ${ }^{20,21}$ Details of the method was given in paper I. The cluster definition follows that of Stillinger, ${ }^{22}$ namely, a group of LJ particles can be defined as a cluster if every particle has at least one nearest neighbor within a distance of less than 1.5 (a value close to the first minimum of the pair correlation function of a LJ liquid near the triple point).

Figure 1 shows the nucleation rate $J$ versus the ratio of the number of carrier-gas particles $N_{c}$ to that of target particles $N_{t}$. For model A (a soft-core type), the nucleation rate increases with increasing the ratio $N_{c} / N_{t}$. The rate for $N_{c} / N_{t}=4$ is an order of magnitude higher than that for $N_{c} / N_{t}=1$. Clearly, with model A, the effect of the carrier-gas pressure is positive. With increasing the number of the carrier-gas particles in the system, the effective volume left for the target particles decreases due to the excluded volume effect caused by the soft-core potential. As a consequence, the actual target density is enhanced, which results in a higher nucleation rate. For model B carrier gas, the nucleation rate is nearly unchanged with increasing the ratio $N_{c} / N_{t}$ for given values $\varepsilon_{\mathrm{cc}}=0.1 \varepsilon$ and $\varepsilon_{\mathrm{cc}}=0.25 \varepsilon$. The effect of the carrier-gas pressure is negligible in this case. For model $\mathrm{C}$ carrier gas, different effects of the carrier-gas pressure were observed. In the case of the weak attractive interaction characterized by $k=0.1$ or 0.3 , the effect of the carrier-gas pressure is positive. On the other hand, in the case of the strong 


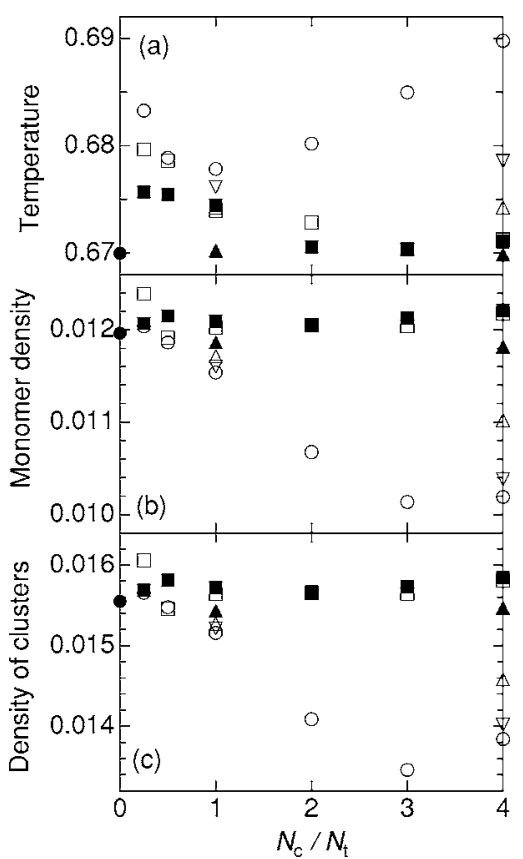

FIG. 2. (a) Average temperature, (b) monomer density, and (c) density of clusters for target particles vs the ratio of the number of carrier-gas particles $N_{c}$ to the number of target particles $N_{t}$ for each MD simulation. The notation is same as in Fig. 1.

attractive interaction characterized by $k=0.5$ the effect of the carrier-gas pressure is negligible. Here, the negative effect due to the stronger attractive interaction between target and carrier-gas particles $(k=0.5)$ offsets the positive effect due to the carrier-gas pressure. Hence, the strength of the attractive interaction between target and carrier-gas particles plays a significant role to the carrier-gas effect.

\section{B. Thermodynamic properties}

Thermodynamic properties of the system were calculated. Specifically, the properpies are the temperature and density of the system as a function of the number of carriergas particles. Figures 2(a)-2(c) show the temperature of target particles, the monomer density (of target gas), and the density of clusters versus the ratio $N_{c} / N_{t}$, respectively. It can be seen from Fig. 2(a) that the temperature of target particles is generally higher than that of carrier-gas particles ( $T=0.67)$, when $N_{c} / N_{t}<1$ for all models. In other words, when the number of carrier-gas particles is small the carrier gas cannot sufficiently release the latent heat generated from nuclei formation. As the number of carrier-gas particles increases towards $N_{c} / N_{t}=1$, the temperature of target particles decreases. For $N_{c} / N_{t} \geqslant 1$, the calculated temperature is dependent on the nucleation rate. If the nucleation rate is high, the temperature of the target gas is higher than that of the carrier gas. If the nucleation rate is low, the temperature of the target gas is almost the same as that of the carrier gas.

Figure 2(b) shows the monomer density of the target gas. The monomer density decreases with increasing the nucleation rate. Moreover, when large clusters are formed, the number of monomers decreases since the total number of target particles is constant $(=40000)$ in the simulations. The density of clusters is defined by $\left\langle\Sigma N_{n_{t}}\right\rangle / V$, ${ }^{23}$ where $n_{t} \geqslant 1$

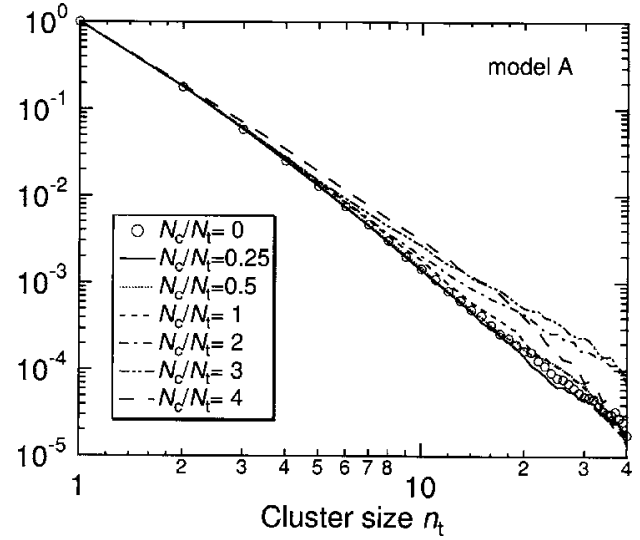

FIG. 3. Cluster distribution for model A or in the case of no carrier gas.

denotes the size of the target cluster and $V$ is the volume of the simulation system. $N_{n_{t}}$ satisfies the condition of the conservation of particles $\sum n_{t} N_{n_{t}}=N_{t}$. The density of clusters is slightly larger than that of the monomer density, indicating that the predominant species is monomers.

\section{Cluster distribution}

Figures 3-5 show the averaged cluster distribution $N_{n_{t}} / N_{1}$ versus $n_{t}$ at the steady state for models A, B, and C, respectively. For model A (shown in Fig. 3) the probability of the cluster increases with increasing $N_{c} / N_{t}$, and so does the nucleation rate. The effect of the carrier-gas pressure is positive. Figures $4(\mathrm{a})$ and 4 (b) show the cluster distribution for the given model B parameter $\varepsilon_{\mathrm{cc}}=0.1$ and $\varepsilon_{\mathrm{cc}}=0.25$, respectively. The cluster distribution is weakly dependent on $N_{c} / N_{t}$ for model B. In Fig. 5, one can see that for $k=0.1$ and $k$ $=0.3$ the probability of the cluster for $N_{c} / N_{t}=4$ is greater

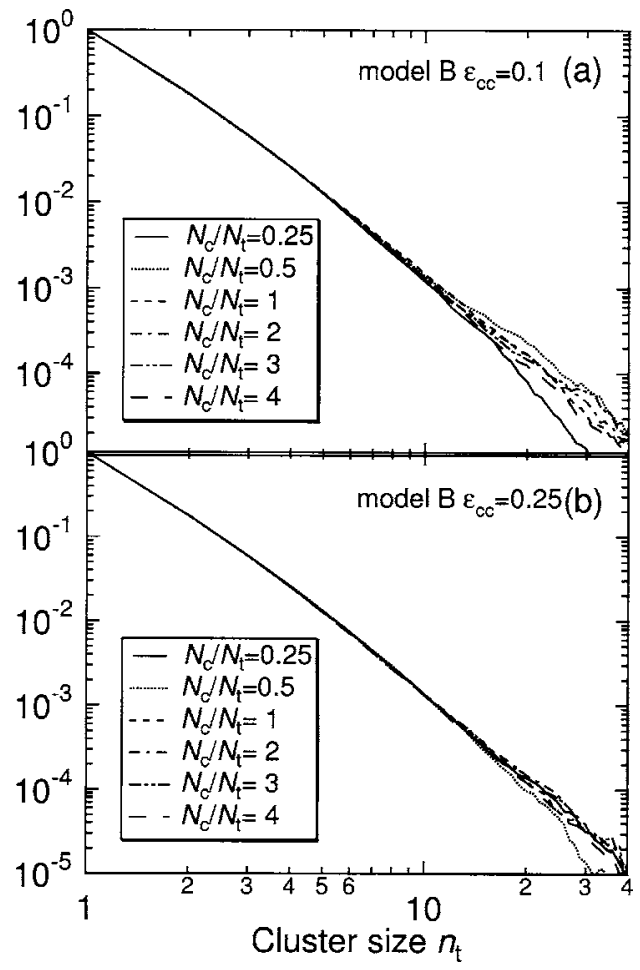

FIG. 4. Cluster distribution for model B: (a) $\varepsilon_{\mathrm{cc}}=0.1$ and (b) $\varepsilon_{\mathrm{cc}}=0.25$. 


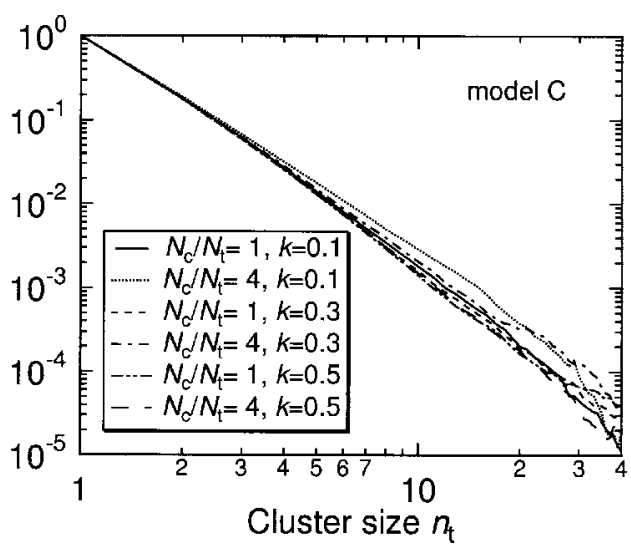

FIG. 5. Cluster distribution for model C.

than that for $N_{c} / N_{t}=1$, showing a positive effect of the carrier-gas pressure. However, for $k=0.5$ the probability of the cluster for $N_{c} / N_{t}=1$ and 4 is nearly same. To summarize, the effect of the carrier-gas pressure based on the results of the cluster distribution is consistent with that based on the calculated nucleation rate.

\section{Behavior of carrier-gas particles near cluster}

\section{Model B}

In order to understand why the effect of the carrier-gas pressure was negligible with model B and negligible or positive with model $\mathrm{C}$, the density profiles of the cluster and that of the carrier gas near the cluster were analyzed. Note that the analysis of simulation results thus far has been focused on the target particles only. In other words, we viewed the nucleation process as a single-component nucleation. The following analysis treats the system as a binary nucleation system.

Figure 6(a) and 6(b) show the density profiles of target particles and carrier-gas particles for $N_{c} / N_{t}=1$ and $\varepsilon_{\mathrm{cc}}=0.1$ (model B). The definition of the cluster is still that of Stillinger, except that the carrier-gas particles are not distinguished from the target particles. The cluster size is defined as the number of target particles in the mixed cluster. For example, $n_{t}=20$ indicates that the number of target particles in the mixed cluster is 20 while some carrier-gas particles are also part of the cluster. In Fig. 6(a) the density profiles of target particles about the center of the cluster are plotted for three cluster sizes. The three density profiles are similar to each other in shape while the cluster volume increases with increasing the cluster size. In Fig. 6(b) the carrier-gas density profiles about the center of the cluster are plotted. It can be seen that the density at the center is an order of magnitude smaller than that of the target density. Interestingly, the peak of the density profile is near the surface of the mixed cluster and its value is comparable to the average density of the carrier gas in the system. This result indicates that the carrier-gas particles tend to be adsorbed near the surface of the target cluster. Hence, the peak of the carrier-gas profile moves outward from the center with increasing the size of the cluster.

Figure 7 shows the density profile for $N_{c} / N_{t}=4$ and $\varepsilon_{\mathrm{cc}}$ $=0.1$. In Fig. 7(a) the density of target particles with $n_{t}=20$

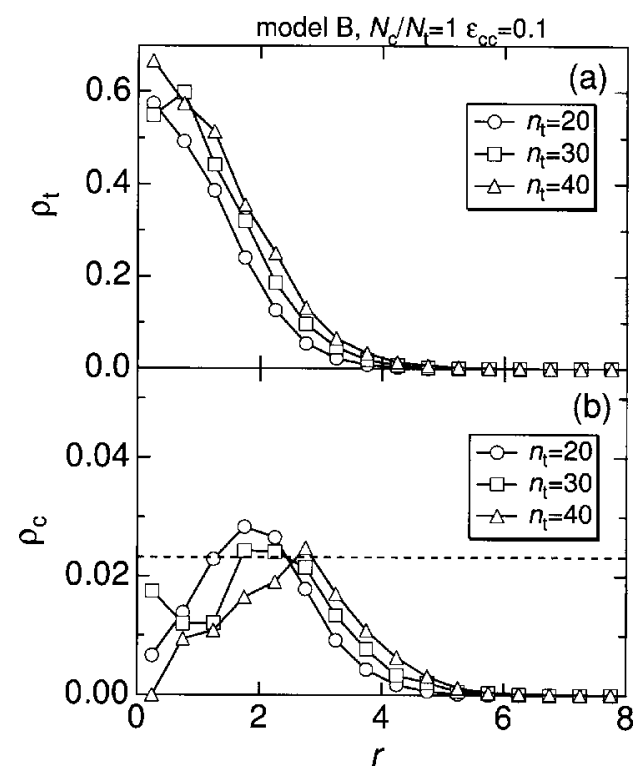

FIG. 6. Density profiles for model B, $\varepsilon_{\mathrm{cc}}=0.1, N_{c} / N_{t}=1$ : (a) target particles and (b) carrier-gas particles. The dotted line is the average density of the carrier gas in the system.

and 30 for $N_{c} / N_{t}=4$ is about a third of that for $N_{c} / N_{t}=1$ (Fig. 6). For $n_{t}=40$, the target profile is about a half of that for $N_{c} / N_{t}=4$. In Fig. 7(b) the density of carrier-gas particles near the core region of the cluster is slightly larger than the average density of carrier-gas particles in the system for $n_{t}=20$ and 30. In all three cases of $n_{t}=20,30$, and 40, the mixed cluster consists of both target and carrier-gas particles as in the case of typical binary nucleation.

Figure 8 shows the density profiles for $N_{c} / N_{t}=1$ and $\varepsilon_{\mathrm{cc}}=0.25$. In Fig. $8(\mathrm{a})$ the target density at the center of the cluster is more or less the same, regardless of the target

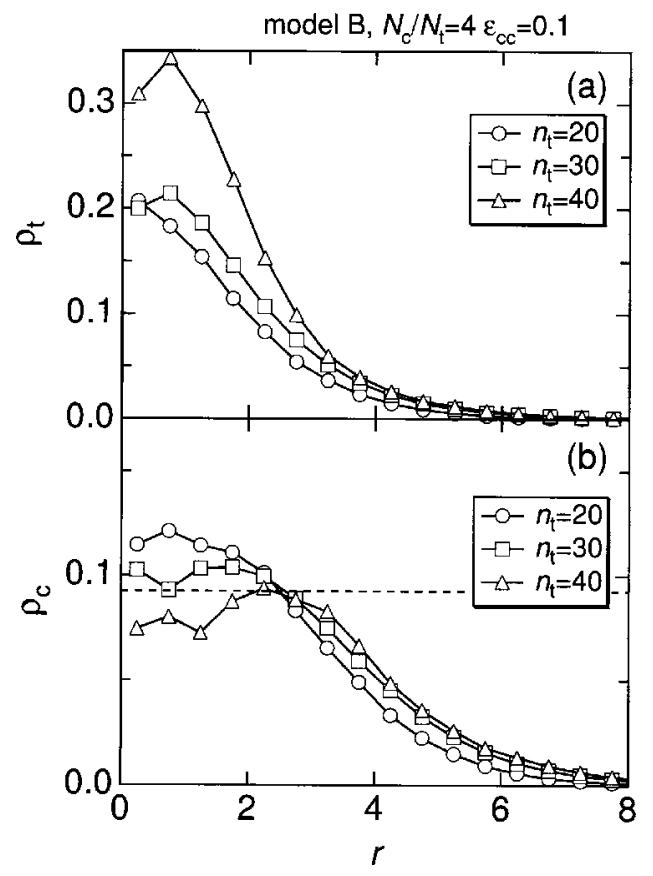

FIG. 7. Density profiles for model B, $\varepsilon_{\mathrm{cc}}=0.1, N_{c} / N_{t}=4$ : (a) target particles and (b) carrier-gas particles. The dotted line is the average density of the carrier gas in the system. 


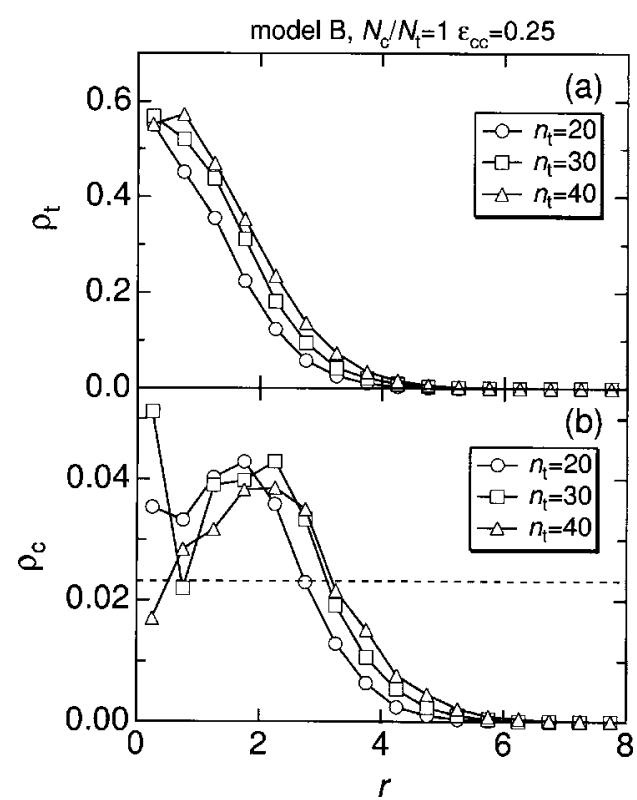

FIG. 8. Density profiles for model B, $\varepsilon_{\mathrm{cc}}=0.25, N_{c} / N_{t}=1$ : (a) target particles and (b) carrier-gas particles. The dotted line is the average density of the carrier gas in the system.

cluster size; the volume of the cluster increases with increasing the cluster size. This behavior is similar to that for $\varepsilon_{\mathrm{cc}}$ $=0.1$ and $N_{c} / N_{t}=1$. In Fig. $8(\mathrm{~b})$ the carrier-gas densities at the center and on the surface of cluster are all larger than the average density of the carrier gas in the system. However, the density of carrier-gas particles is still an order of magnitude smaller than that of target particles. Most of the carriergas particles in the mixed cluster are adsorbed on the surface of the cluster, similar to the case of $\varepsilon_{\mathrm{cc}}=0.1$ and $N_{c} / N_{t}=1$.

Figure 9 shows the density profiles for $N_{c} / N_{t}=4$ and $\varepsilon_{\mathrm{cc}}=0.25$. In Fig. 9(a) the target density at the center of the

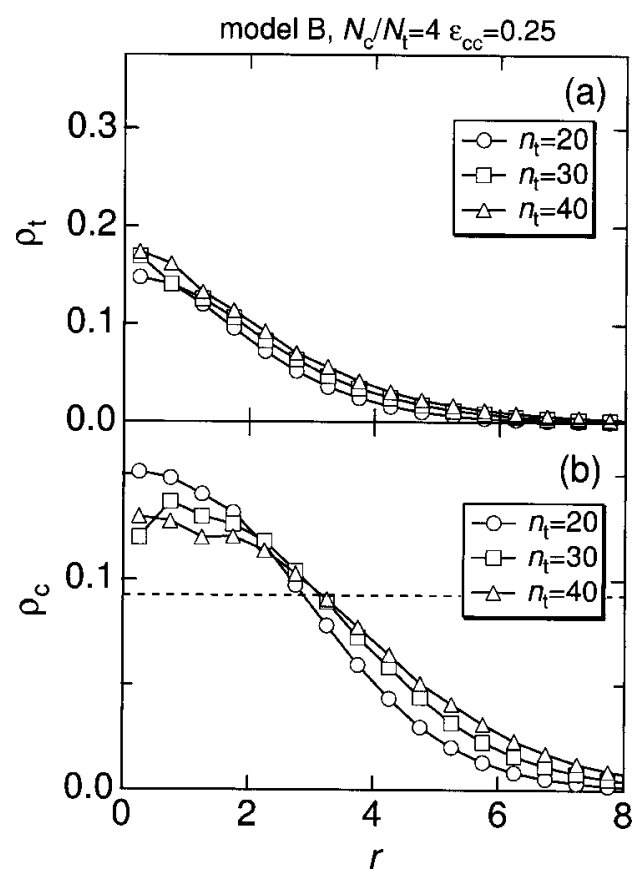

FIG. 9. Density profiles for model B, $\varepsilon_{\mathrm{cc}}=0.25, N_{c} / N_{t}=4$ : (a) target particles and (b) carrier-gas particles. The dotted line is the average density of the carrier gas in the system.

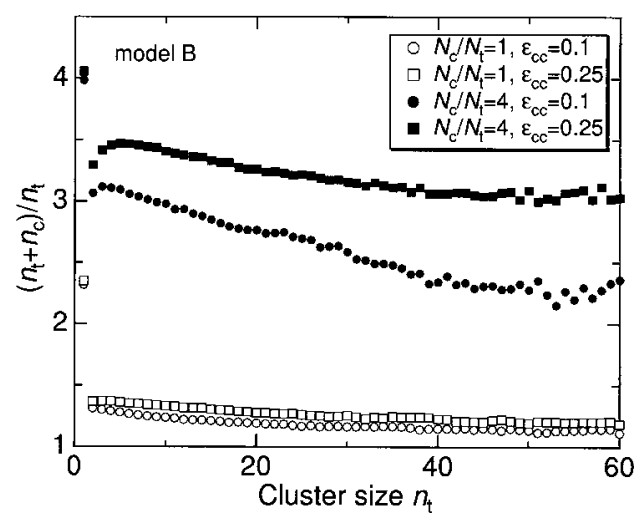

FIG. 10. Ratio of the binary cluster size $n_{t}+n_{c}$ to the cluster size $n_{t}$, where $n_{c}$ is the number of carrier-gas particles in the cluster for model B.

cluster is also more or less the same. The density of target particles is a third of that for $\varepsilon_{\mathrm{cc}}=0.1$ and $N_{c} / N_{t}=4$. In Fig. 9(b) the carrier-gas densities at the center and on the surface of cluster are larger than the average density of the carrier gas in the system. The mixed cluster contains a comparable number of target and carrier-gas particles, when the ratio $N_{c} / N_{t}=4$. Since the mixed cluster can be viewed as a cluster of binary nucleations, we also estimated the number of the carrier-gas particles in each cluster. Figure 10 shows the ratio of the binary-cluster size to the (target) cluster size, $\left(n_{t}+n_{c}\right) / n_{t}$, where $n_{t}$ is defined as the number of target particles in the cluster and $n_{c}$ is the number of carrier-gas particles in the cluster. For $N_{c} / N_{t}=1$ the number of carrier-gas particles in the cluster is relatively small in each case of $\varepsilon_{\mathrm{cc}}$.

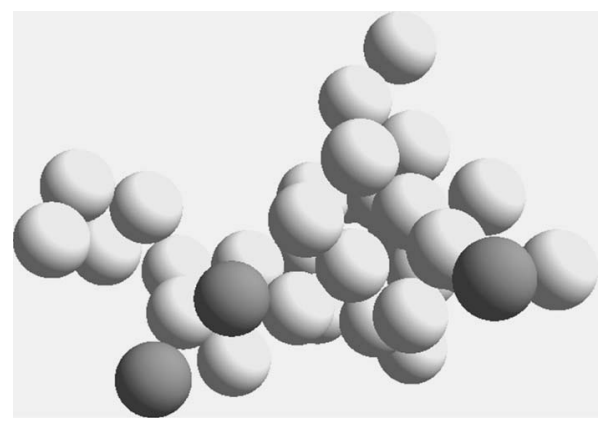

(a)

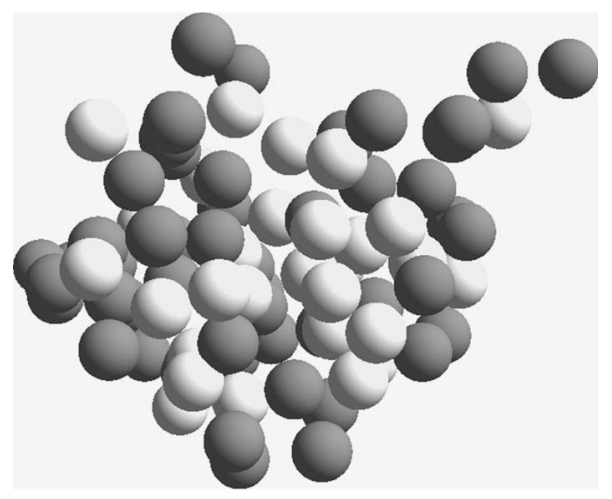

(b)

FIG. 11. A typical snapshot of the cluster $\left(n_{t}=30\right)$ for model $\mathrm{B}, \varepsilon_{\mathrm{cc}}=0.25$ : (a) $N_{c} / N_{t}=1$ and (b) $N_{c} / N_{t}=4$. Light spheres represent the target particle and dark spheres represent the carrier-gas particle. 


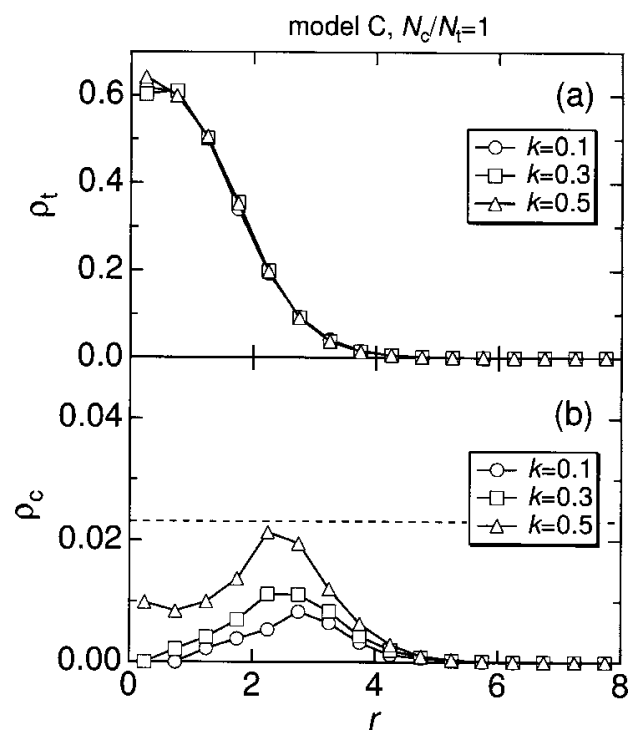

FIG. 12. Density profiles of model C for $N_{c} / N_{t}=1$ : (a) target particles and (b) carrier-gas particles.

So the ratio is close to 1 . For $N_{c} / N_{t}=4$ and $\varepsilon_{\mathrm{cc}}=0.1$ the ratio is about two to three, indicating that the the mixed cluster contains a considerable amount of carrier-gas particles. For $N_{c} / N_{t}=4$ and $\varepsilon_{\mathrm{cc}}=0.25$, the ratio is even larger, and the total binary-cluster size is more than three times of $n_{t}$.

Figure 11 displays snapshots of the mixed clusters with $n_{t}=30$ for $N_{c} / N_{t}=1$ and $N_{c} / N_{t}=4$, respectively. In Fig. 11(a) (for $N_{c} / N_{t}=1$ ), the carrier-gas particles (dark spheres) are adsorbed on the surface of the target cluster (light spheres). Since the cluster was not in spherical shape, the density of carrier-gas particles at the center of the cluster was actually not zero. In Fig. 11(b) (for $N_{c} / N_{t}=4$ ), the cluster is well mixed with both the target and carrier-gas particles.

\section{Model C}

The density profiles were also calculated to examine the effect of the attractive interaction, characterized by the $k$ pa-

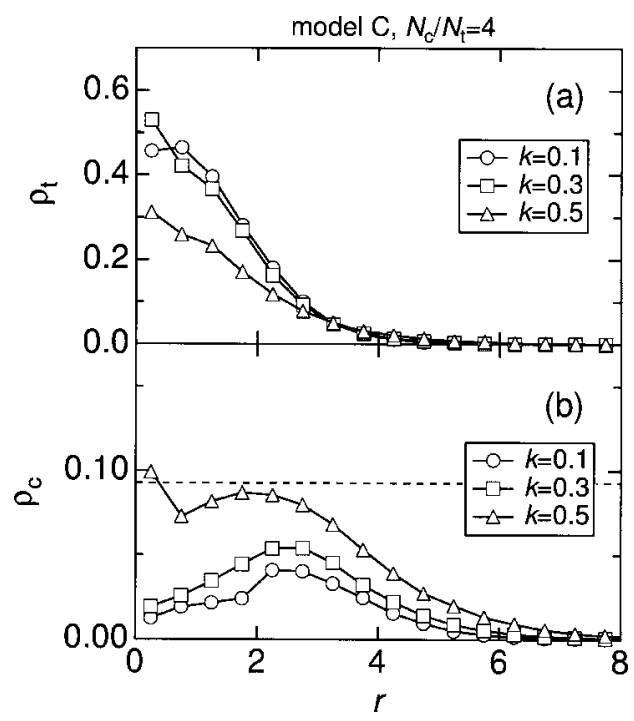

FIG. 13. Density profiles of model C for $N_{c} / N_{t}=4$ : (a) target particles and (b) carrier-gas particles.

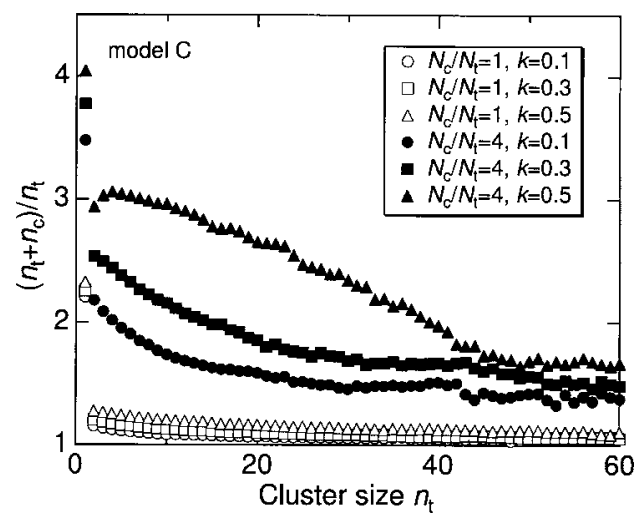

FIG. 14. Ratio of the binary cluster size $n_{t}+n_{c}$ to the cluster size $n_{t}$, where $n_{c}$ is the number of carrier-gas particles in the cluster for model C.

rameter in model $\mathrm{C}$, to the nucleation. Figure 12 shows the density profiles for $N_{c} / N_{t}=1$. In Fig. 12(a) the target density profiles are nearly the same for the three given parameter $k$. In Fig. 12(b) the carrier-gas densities at the center and on the surface of cluster are less than the average density of carrier gas in the system. Moreover, the carrier-gas densities are an order of magnitude smaller than the target densities. The carrier-gas particles adsorbed on the surface of the cluster increase with increasing the $k$ parameter. In other words, if the attractive interaction is relatively stronger, the number of adsorbed carrier-gas particles increases.

Figure 13 shows the density profiles for $N_{c} / N_{t}=4$. For $k=0.1$ and 0.3 the density profiles of target particles are similar to those of model B for $N_{c} / N_{t}=1$ (see Fig. 8). The carriergas particles are mostly adsorbed on the cluster surface. However, for $k=0.5$ the density profiles are similar to those of model B for $N_{c} / N_{t}=4$ (see Fig. 7). The cluster is well mixed with both target and carrier-gas particles. In other words, the stronger the attractive interaction between carriergas and target particles, the cluster behaves more like a binary cluster.

Figure 14 shows the ratio $\left(n_{t}+n_{c}\right) / n_{t}$ versus $n_{t}$ for model C carrier gas. For $N_{c} / N_{t}=1$, the number of carrier-gas particles in the cluster is very small, regardless of the $k$ value. This result is similar to that for model B. For $N_{c} / N_{t}=4, k$ $=0.1$ and $k=0.3$, the ratio $\left(n_{t}+n_{c}\right) / n_{t}$ ranges from about 1.5 to 2 , indicating that the target particles are still the dominant component in the mixed cluster. However, for $k=0.5$, the binary-cluster size is much larger than the (target) cluster size for $n_{t}<40$, indicating that the dominant component is the carrier-gas particles. The cluster behaves like a binary cluster.

\section{CONCLUSION}

We have carried out molecular dynamics simulations of vapor mixtures with 40000 target particles and 0-160 000 carrier-gas particles to investigate the effect of the carrier-gas pressure to nucleation. A Lennard-Jones potential was adopted for the target particles and three potential models were adopted for the carrier-gas particles. The first model, model A, is a soft-core potential as used in the previous study. $^{20}$ The second model, model B, is a Lennard-Jones po- 
tential, and the third, model $\mathrm{C}$, is a modified Lennard-Jones potential in which the attractive interaction can be adjusted.

We find that the effect of the carrier-gas pressure to the nucleation is positive for model A but negligible for model B. For model C with a weak attractive interaction $(k=0.1$ and 0.3 ), the carrier-gas effect is positive but with $k=0.5$ the effect is negligible. To understand the carrier-gas effect with models B and C, we carefully analyzed the density profiles of both target and carrier-gas particles of the clusters. With model $\mathrm{B}$, it is found that the carrier-gas particles are adsorbed on the cluster surface when the ratio $N_{c} / N_{t}=1$. For larger ratio $N_{c} / N_{t}=4$, the carrier-gas particles are well mixed in the cluster. In both cases, however, the effect of the carrier-gas pressure is negligible. With model $\mathrm{C}$ and for $N_{c} / N_{t}=1$, the carrier-gas particles are adsorbed on the cluster surface. For a larger ratio $N_{c} / N_{t}=4$ and a weaker attractive interaction $k=0.1$ and 0.3 , the carrier-gas particles are also adsorbed on the cluster surface. However, for $N_{c} / N_{t}=4$ and a stronger attractive interaction $k=0.5$, the carrier-gas particles are well mixed in the cluster. From these results, we conclude that the attractive interaction between target and carrier-gas particles is mainly responsible to the negative effect of the carrier gas whereas the repulsive interaction is mainly responsible to the positive effect of the carrier gas. In the case of model B as well as in the case of $k=0.5$ of model $\mathrm{C}$ the positive effect of the carrier gas is offset by the negative effect, resulting in a negligible effect of the carrier-gas pressure.

\section{ACKNOWLEDGMENTS}

The authors are gratefule for valuable discussions with Dr. S. Yoo. This work is supported by grants from the DOE's Office of Basic Energy Sciences (DE-FG02-04ER46164),
National Science Foundation (CHE-0427746), the John Simon Guggenheim Foundation, the Nebraska Research Initiative, and the UNL Research Computing Facility.

${ }^{1}$ J. L. Katz, J. A. Fisk, and V. M. Chakarov, in Nucleation and Atmospheric Aerosols, edited by N. Fukuta and P. E. Wagner (Deepak, Hampton, 1992).

${ }^{2}$ R. H. Heist, M. Janjua, and J. Ahmed, J. Phys. Chem. 98, 4443 (1994).

${ }^{3}$ R. H. Heist, J. Ahmed, and M. Janjua, J. Phys. Chem. 99, 375 (1995).

${ }^{4}$ A. Bertlsmann, R. Stuczynski, and R. H. Heist, J. Phys. Chem. 100, 9762 (1996).

${ }^{5}$ D. Kane and M. S. El-Shall, J. Chem. Phys. 105, 7617 (1996).

${ }^{6}$ G. Wilemski, B. E. Wyslouzil, M. Gauthier, and M. B. Frisk, in Nucleation and Atmospheric Aerosols, edited by N. Fukuta and P. E. Wagner (Deepak, Hampton, 1992).

${ }^{7}$ P. E. Wagner, R. Strey, and Y. Viisanen, in Nucleation and Atmospheric Aerosols, edited by N. Fukuta and P. E. Wagner (Deepak, Hampton, 1992).

${ }^{8}$ Y. Viisanen, R. Strey, and H. Reiss, J. Chem. Phys. 99, 4680 (1993).

${ }^{9}$ Y. Viisanen and R. Strey, J. Chem. Phys. 101, 7835 (1994).

${ }^{10}$ J. A. Fisk and J. L. Katz, J. Chem. Phys. 104, 8649 (1996).

${ }^{11}$ D. Brus and V. Zdímal, J. Chem. Phys. 124, 164306 (2006).

${ }^{12}$ A.-P. Hyvärinen, D. Brus, V. Zdímal, J. Smolík, M. Kulmala, Y. Viisanen, and H. Lihavainen, J. Chem. Phys. 124, 224304 (2006).

${ }^{13}$ C. C. M. Luijten, K. J. Bosschaart, and M. E. H. van Dongen, J. Chem. Phys. 106, 8116 (1997).

${ }^{14}$ C. C. M. Luijten, P. Peeters, and M. E. H. van Dongena, J. Chem. Phys. 111, 8535 (1999).

${ }^{15}$ M. P. Anisimov, P. K. Hopke, S. D. Shandakov, and I. I. Shvets, J. Chem. Phys. 113, 1971 (2000).

${ }^{16}$ K. J. Oh and X. C. Zeng, J. Chem. Phys. 114, 2681 (2001).

${ }^{17}$ K. K. Tanaka, K. Kawamura, H. Tanaka, and K. Nakazawa, J. Chem. Phys. 122, 184514 (2005).

${ }^{18}$ H. Y. Tang and I. J. Ford, J. Chem. Phys. 125, 144316 (2006).

${ }^{19}$ S. Toxvaerd, J. Chem. Phys. 119, 10764 (2003).

${ }^{20}$ K. Yasuoka and M. Matsumoto, J. Chem. Phys. 109, 8451 (1998).

${ }^{21}$ K. Yasuoka and M. Matsumoto, J. Chem. Phys. 109, 8463 (1998).

${ }^{22}$ F. H. Stillinger, J. Chem. Phys. 38, 1486 (1963).

${ }^{23}$ K. J. Oh and X. C. Zeng, J. Chem. Phys. 110, 4471 (1999). 